\section{Blastocystis hominis: o enigma continua}

\author{
Blastocystis hominis: the enigma goes on
}

Ao Editor: Blastocystis hominis é um protozoário freqüentemente encontrado nas fezes de pessoas com e sem manifestações gastrintestinais, imunocompetentes e imunossuprimidos 18111516 19. De todos os microorganismos possíveis de serem observados nas fezes, Lee considera que $B$. hominis é o segundo mais freqüente, somente superado pelas leveduras ${ }^{11}$. Em nossa experiência, o protozoário é o parasito mais comum, segundo os resultados dos exames coprológicos feitos no ano 1996 no Laboratório de Parasitologia do Departamento de Parasitologia e Microbiologia da Universidade de Oriente, Venezuela (R Devera: dados não publicados).

Blastocystis hominis foi descrito há quase 80 anos, mas ainda persistem controvérsias e até aspectos enigmáticos sobre este parasito intestinal 1920 , sendo sua localização taxonômica e patogenicidade as principais.

Recentemente, no XXXIV Congresso da Sociedade Brasileira de Medicina Tropical, foi reportada uma prevalência de $2,94 \%$ para $B$. hominis numa amostra de indivíduos de São Paulo, ressaltando a utilidade do exame direto de fezes e a coloração com tionina no diagnóstico ${ }^{14}$. Também, recentemente, um estudo no Japão demonstrou uma prevalência de apenas $0,5 \%$ nesse pais 7 . Na Venezuela, nós também empregando a técnica de exame direto como "Gold Standart Test", determinamos uma taxa de prevalência de $17,5 \%$ num grupo de 705 crianças entre 3 e 14 anos de duas creches e sete escolas do Estado Bolívar (R Devera: dados não publicados).

Estas diferenças tão grandes de uma região a outra sem duvida obedecem a fatores geográficos e a fatores dependentes do parasito que ainda não conhecemos. Porém, também devem-se, em parte, ao desconhecimento que há sobre o protozoário, além de que sua identificação requer certa experiência. Em muitos locais o parasito não é reportado rotineiramente nos resultados dos exames coproparasitológicos, além de que muitos o consideram como um organismo um comensal sem importância, esquecendo o número crescente de publicações que o assinalam como parasito potencial em ausência de outras causas que expliquem as manifestações clínicas nos pacientes2 111215161718 .
Recentemente, realizamos uma revisão sobre o protozoário no Brasil, e somente foram encontrados cinco artigos onde o parasito foi estudado, ou pelo menos foi mencionado 168913.

Nos sugerimos que $B$. hominis é um organismo com uma morfologia única, porém com constituição bioquímica e genética diversas, que redunda em potencialidades patogênicas diferentes. Poderia tratar-se de um complexo de espécies, o qual explicaria as diferenças tão marcadas na patogenia e epidemiologia que são observadas de uma área geográfica para outra.

Diferentes trabalhos citados em muitos jornais, parecem indicar que essa sugestão é correta. Em 1991, Kukoschke e Muller detectaram cepas de $B$. hominis bioquímica e imunologicamente diferentes em cultivos axênicos. Pelo menos duas variantes com diferentes padrões polipeptídicos e antigênicos foram demonstradas pelos pesquisadores ${ }^{10}$. Gericke et al3 identificaram cinco padrões isoenzimáticos em isolados de $B$. hominis procedentes de pacientes sintomáticos e portadores sadios, embora não encontrassem correlação entre esses padrões e a doença dos pacientes. Essas observações levaram a outros autores a empregar técnicas de biologia molecular para determinar se com elas haveria diferenças. Assim, cinco grupos foram evidenciados, tanto em pacientes assintomáticos como sintomáticos ${ }^{4}$. Graham-Clark empregando riboprinting para estudar ao parasito, demonstrou que $B$. hominis tem ampla diversidade genética, - que poderia explicar a disparidade de observações encontradas em relação à patogenia do protozoário5 .

A falta de correlação entre os grupos determinados e a clínica dos pacientes, indica que possivelmente requer-se estudar maior número de pacientes de diversas procedências geográficas.

Entretanto, com esses dados, a questão mais importante ainda não foi resolvida: $B$. hominis e um patógeno ou um comensal? Estudos clinicoepidemiologicos baseados nas novas ferramentas da medicina atual serão fundamentais nos próximos anos para dar resposta a tão difícil pergunta que continua sendo uma das maiores incógnitas de um parasito enigmático: $B$. hominis.

1. Brites C, Barberino G, Bastos M A, Sá MS, Silva N. Blastocystis hominis as a potential cause of diarreha in AIDS 
patients: a report of six cases in Bahia, Brazil. The Brazilian Journal of Infectious Disease 1:91-94, 1997.

2. Carrascosa M, Martínez J, Pérez-Castrillón JL. Hemorrhagic proctosigmoiditis and Blastocystis hominis infection. Annals of Internal Medicine 124:278-179, 1996.

3. Gericke AS, Burchard GD, Knobloch J, Walderich B. Isoenzyme patters of Blastocystis hominis patient isolates derived from symptomatic and healthy carriers. Tropical Medicine and International Health 2:245-253, 1997

4. Gloning BB, Knobloch J, Walderich B. Five subgrupes of Blastocystis hominis isolates from symtomatic and asymptomatic patients reveled by restriction site analysis of PCR-amplified 16S-like rDNA. Tropical Medicine and International Health 2:771-778, 1997.

5. Graham-Clarck G. Extensive genetic diversity in Blastocystis hominis Molecular and Biochemical Parasitology 87:79-83, 1997.

6. Guimaraes S, Sogayar MI.Blastocystis hominis: Ocurrence in children and staff membres of municipal day-care centers from Botucatu, São Paulo State, Brazil. Memórias do Instituto Oswaldo Cruz 88:427-429, 1993.

7. Horiki N, Maruyama M, Fujita J, Yonekura T, Minato S, Kaneda Y. Epidemiologic survey of Blastocystis hominis infection in Japan. Americam Journal of Tropical Medicine and Hygiene 56:370-374, 1997.

8. Jones TC. Blastocystis hominis: More than 70 years of debate regarding pathogenicity! The Brazilian Journal of Infectiouns Disease 1:102-104, 1997.

9. Kobayashi J. Hasegaw H, Forli AA. Prevalence of intestinal parasitic infection in five farms in Holambra, São Paulo, Brazil. Revista do Instituto de Medicina Tropical de São Paulo 37:13-18, 1995.

10. Kukoschke KG, Muller HE. SDS-PAGE and immunological analysis of different axenic Blastocystis hominis. Journal of Medical Microbiology 35:35-39, 1991.

11. Lee MJ. Pathogenicity of Blastocystis hominis. Journal of Clinical Microbiology 29:2089, 1991.

12. Markell EK, Udkow MP. Blastocystis hominis pathogen or fellow traveller? Americam Journal of Tropical Medicine and Hygiene 35:1023-1026, 1986.

13. Moura H, Fernandez O, Viola JPB, Silva SP, Passos RH, Lima DB. Enteric parasites and HIV infection: Occurrence in AIDS patient in Rio de Janeriro, Brazil. Memórias do Instituto Oswaldo Cruz 84:527-533, 1989.

14. Pires de Mato C, Amato Neto V, Braz LMA, Carignani FL, Villela MSH, Pinto THL, Di Pietro Fernandes AO, De Marchi Casadei RM. Blastocystis hominis. Diagnóstico por exame direto e por coloração com tionina. Revista da Sociedade Brasileira de Medicina Tropical 31 (supl 1):188, 1998.

15. Ponce de León P, Svetaz M J, Zdero M. Importancia del diagnóstico de Blastocystis hominis en el examen parasitológico de heces. Revista Latino-americana de Microbiología 33:159-164, 1991

16. Sheehan DJ, Raucher BG, Mckitrick JC. Association of Blastocystis hominis with signs and simptoms of human disease. Journal of Clinical Microbiology 24:548-550, 1986.

17. Sinniah B, Rajeswari B. Blastocystis hominis intection, a cause of diarrhea. Southeast Asian Journal of Tropical Medicine and Public Health 25:490-493, 1994.
18. Udkow MP, Markell E K. Blastocystis hominis: Prevalence in Asymptomatics versus Symptomatics hosts. Journal of Infectious Disease 168:242-244, 1993.

19. Zierdt $\mathrm{CH}$. Blastocystis hominis-Past and future. Clinical Microbiology Reviews 4:61-79, 1991.

20. Zierdt CH, Rude W S, Bull BS. Protozoan characteristics of Blastocystis hominis. Americam Journal of Clinical Pathology 48:495-501,1967.

\section{Rodolfo Devera}

Departamento de Parasitologia e Microbiologia, Universidade de Oriente, Ciudad Bolívar, Venezuela. Departamento de Medicina Tropical, Instituto Oswaldo Cruz, FIOCRUZ. Rio de Janeiro, RJ, Brasil.

Recebido em 21/05/98.

\section{Seria a taxonomia insignificante para a ecologia de vetores?}

\section{Would taxonomy be insignificant to the vector's ecology?}

Ao Editor: A correta identificação dos vetores potenciais de parasitos é fundamental para a compreensão de seu possível papel na transmissão. A imprecisão na identificação destes insetos nas várias regiões, desconsiderando os estudos taxonômicos, pode levar a confusão entre espécies vetoras e não vetoras e com características biológicas diferentes. É freqüente que uma espécie seja descrita e, após estudos mais detalhados, esta se revele suficientemente heterogênea para ser dividida em entidades mais homogêneas e menos abrangentes. Isto é mais evidente e fundamental para certos anofelíneos e simulídeos, que constituem complexos de espécies, em que as várias espécies componentes apresentam, por exempo, diferenças nas preferências alimentares e no papel vetorial2.

Estudos recentes têm indicado a existência de tais complexos em Phlebotominae, especialmente em Lutzomyia longipalpis (Lutz \& Neiva, 1912) 313 , L. intermedia (Lutz \& Neiva, $1912)^{4} 5$ e L. Whitmani (Antunes \& Coutinho, 1939)9. O estudo morfométrico detalhado de 705 exemplares de L. intermedia s. I. procedentes de 12 estados do Brasil e do Paraguai, Argentina e Bolívia, além do estudo da genitália de vários outros do Estado de São Paulo, permitiu distinguir duas espécies bem definidas, L. intermedia S.S. e L. neivai (Pinto, 1926)4 5, assim como definir a distribuição geográfica do complexo e das duas espécies 6 . 
No entanto, têm sido publicados trabalhos de ecologia de flebotomíneos, que citam o encontro de $L$. intermedia na região do Estado de São Paulo ao oeste da Serra do Mar1 8 e nos Estados do Paraná11 12 e do Rio Grande do Sul10. Nestas regiões, todos os numerosos exemplares estudados foram identificados como pertencentes a L. neivai 4567 .

É muito importante que, no futuro, os autores de tais trabalhos e de outros correlatos a serem publicados, mesmo que apenas relacionados com a ecologia de flebotomíneos e com a epidemiologia de leishmanioses, divulguem a identificação específica mais precisa dos insetos capturados. Se discordarem da proposta de distinção das duas espécies, esta discordância deve ser explicitamente referida. Outra alternativa viável, mas menos precisa, seria a de citarem a identificação dos exemplares como L. intermedia s.l.

1. Condino MLF, Sampaio SMP, Henriques LF, Galati EAB, Wanderley DMV, Corrêa FMA. Leishmaniose tegumentar americana: flebotomíneos de área de transmissão no Município de Teodoro Sampaio, região sudoeste do Estado de São Paulo, Brasil. Revista da Sociedade Brasileira de Medicina Tropical 31:355-360, 1998.

2. Lane RP, Crosskey, RW. General introduction. In: Lane RP, Crosskey RW (ed) Medical insects and arachnids. Chapman \& Hall, London, p.1-29, 1993.

3. Lanzaro GC, Ostrovska K, Herrero MV, Lawyer PG, Warburg A. Lutzomyia longipalpis is a species complex divergence and interspecific sterility among three populations. American Journal of Tropical Medicine and Hygiene 48:839-847, 1993.

4. Marcondes CB. A redescription of Lutzomyia (Nyssomyia) intermedia (Lutz \& Neiva, 1912) and resurrection of L. neival (Pinto, 1926) (Diptera Psychodidae, Phlebotominae). Memórias do Instituto Oswaldo Cruz 91:457-462, 1996.

5. Marcondes CB. Morfometria e DNA mitocondrial de populações sul americanas de Lutzomyia (Nyssomyia) intermedia (Lutz \& Neiva, 1912) (Diptera Psychodidae, Phlebotominae). Tese de Doutorado. Curitiba, Universidade Federal do Paraná. xxiv+260p. 1997.

6. Marcondes CB. Morfometria e DNA mitocondrial de populações americandas de Lutzomyia (Nyssomyia) intermedia (Lutz \& Neiva, 1912) (Diptera Psychodidae, Phlebotominae). Revista da Sociedade Brasileira de Medicina Tropical 30:533-534, 1997.

7. Marcondes CB, Lozovei AL, Vilela JH. Distribuição geográfica de flebotomíneos do complexo Lutzomyia intermedia (Lutz \& Neiva, 1912). Revista da Sociedade Brasileira de Medicina Tropical 31:51-58, 1998.

8. Mayo RC, Casanova C, Mascarini LM, Pignatti MG, Rangel $O$, Galati EAB, Wanderley DMV, Corrêa FMA. Flebotomíneos (Diptera Psychodidae) de área de transmissão de leishmaniose tegumentar americana, no Município de Itupeva, região sudeste do Estado de São Paulo, Brasil. Revista da Sociedade Brasileira de Medicina Tropical 31:339-345, 1998

9. Ready PD, Day JC, Souza AA, Rangel EF, Davies CR. Mitochondrial DNA characterization of populations of Lutzomyia whitmani (Diptera: Psychodidae) incriminated in the peri-domestic and silvatic transmission of Leishmania species in Brazil. Bulletin of Entomological Research 87:187195, 1997.

10. Silva OS, Grunewald J. Preliminary studies on Phlebotomine fauna from Parque Estadual do Turvo, RS, an endemic tegumentary leishmaniasis area. Memórias do Instituto Oswaldo Cruz 92 (supl I):317, 1997.

11. Teodoro U, Kühl JB. Interação flebotomíneos, animais domésticos e dominância de Lutzomyia (Nyssomyia) intermedia (Lutz \& Neiva, 1912) em área com alto grau de antropia, no sul do Brasil. Revista de Saúde Pública 31:512516, 1997.

12. Teodoro U, Kühl JB, Thomaz-Soccol V, Barbosa OC, Ferreira MEMC, Lozovei AL, Silveira TGV. Influence of peri-domiciliar environmental conditions in the dispersion and proliferation of Phlebotomine sandflies in Paraná State, Southern Brazil. Arquivos de Biologia e Tecnologia 40:745-751, 1997.

13. Ward RD, Philips A, Burnet B, Marcondes CB. The Lutzomyia longipalpis complex: reproduction and distribution. In: Service M (ed) Biosystematics of haematophagous insects. Claredon Press, Oxford, 1988.

\section{Carlos Brisola Marcondes}

Departamento de Microbiologia e Parasitologia.

Centro de Ciências Biológicas da Universidade Federal de Santa Catarina, Florianópolis, SC, Brasil

Recebido em 29/07/98 